\title{
Spontaneous tumor lysis syndrome in breast cancer: a case report and discussion
}

\author{
Ahmed Elfar, Marwa Teleb, Carlos E Rodriguez-Castro and Zeina Nahleh* \\ *Correspondence: zeina.nahleh@ttuhsc.edu \\ Texas Tech University Health Science Center-Paul L Foster School of Medicine, El Paso, TX, USA.
}

\begin{abstract}
Tumor lysis syndrome (TLS) refers to the constellation of metabolic disturbances manifested by acute renal failure associated with marked hyperuricemia. TLS may be seen after initiation of cancer treatment. It usually occurs in patients with bulky, rapidly proliferating, treatment-responsive tumors. Spontaneous TLS, occuring prior to the initiation of cancer therapy, has been reported in hematologic malignancies like high grade non Hodgkin's Lymphoma (NHL) and acute leukemia, and in at least one patient with inflammatory breast cancer. The actual incidence of this occurrence is difficult to ascertain. We present, in this report, the case of a woman with a stage IV adenocarcinoma of the breast diagnosed with spontaneous TLS, and we review the current literature on this rare but interesting disease manifestation.
\end{abstract}

Key words: Tumor, breast, adenocarcinoma, tumor lysis, syndrome, spontaneous

\section{Case presentation}

The patient is a 46 year old woman with a history of stage IV breast cancer diagnosed one year prior to her presentation to our hospital. The metastatic cancer was manifested by metastatic pulmonary nodules, hepatic metastasis, and extensive bony metastasis. A biopsy of the liver in another facility revealed adenocarcinoma positive for estrogen and progesterone receptors, AE1/AE3, CK7, CEA, CA 19.9, P53, and GCDFP-15. She was prescribed tamoxifen, but she has not taken the medicine. The patient has not received any other treatment for her cancer and was lost to follow up.

During this hospitalization, she presented with fatigue, tiredness, nausea, multiple episodes of vomiting, abdominal pain, and diarrhea of one week duration. She was treated empirically with antibiotics, as outpatient, with no relief. Her symptoms increased 3 days prior to the hospitalization and progressively worsened over time. She described the diarrhea as yellow watery stool associated with blood and the vomiting as yellow colored fluids. She denied tenesmus. She denied intake of herbal supplements or other medications.

Review of her medical history was significant for hospitalization for hypercalcemia and acute kidney injury, 2 months prior to this current hospitalization, which resolved with intravenous hydration. Her previous surgical history includes a Cesarean section; and her family history was significant for coronary artery disease. Her physical exam was only significant for pale skin and tachycardia with pulse of 102 beats/minute and diffuses abdominal tenderness. Her laboratory results were significant for: Normocytic anemia with hemoglobin (HGB) $11.1 \mathrm{~g} / \mathrm{dl}$ [normal 12-15], hematocrit (HCT) 32.1\% [normal 36-47], elevated liver enzymes: Aspartate Aminotransferase (AST) 316 Intern Unit/L [normal 12-45], Alkaline phosphatase 863 Intern Unit/L [normal 37-107], low Sodium 123 mmol/L [normal 135-145], calcium 7.9 MG/DL [normal 8.4-10.2], elevated potassium $5.6 \mathrm{mmol} / \mathrm{L}$ [normal 3.9-5.3], elevated phosphorus 5.5 MG/DL [normal 2.4-4.7], elevated BUN $182 \mathrm{MG} / \mathrm{DL}$ [normal 7-22], and creatinine13.34 MG/DL [normal 0.5-1.2], elevated serum uric acid 27.9 MG/DL [normal 1.9-8.2, morning CORTISOL was $34.1 \mathrm{mcg} / \mathrm{dl}$ [normal AM Level 6.7-22.6]. Her fecal occult blood test was positive, and $\mathrm{C}$-difficile toxin was negative. Her urine analysis showed trace proteinuria, blood, 10-15 RBCS, but was negative for casts or crystals. The urine PH was 6 .

\section{Computer tomography scan (CT) of abdomen}

Showed multiple hypodense lesions in the liver which represent her metastatic disease; innumerable lytic lesions involving the spine, ribs, sacrum, pelvis and femurs; and multi level vertebral compression fracture deformity in the lower thoracic and in L2 and L3 vertebral bodies (Figure 1A,1B).

\section{Hospitalization course}

The patient was admitted to the hospital with a clinical diagnosis of: 1) stage IV Breast Cancer, 2) dehydration due to vomiting and diarrhea, 3) normocytic anemia due to acute blood loss versus anemia of chronic disease, 4) acute kidney injury possibly due to tumor lysis syndrome, 5) hyponatraemia, 6) bloody diarrhea due to infectious causes like Clostridium difficile(C Dif) colitis versus tumor. The patient was diagnosed with tumor lysis syndrome, in view of hyperkalemia (despite diarrhea), hyperphosphatemia, 

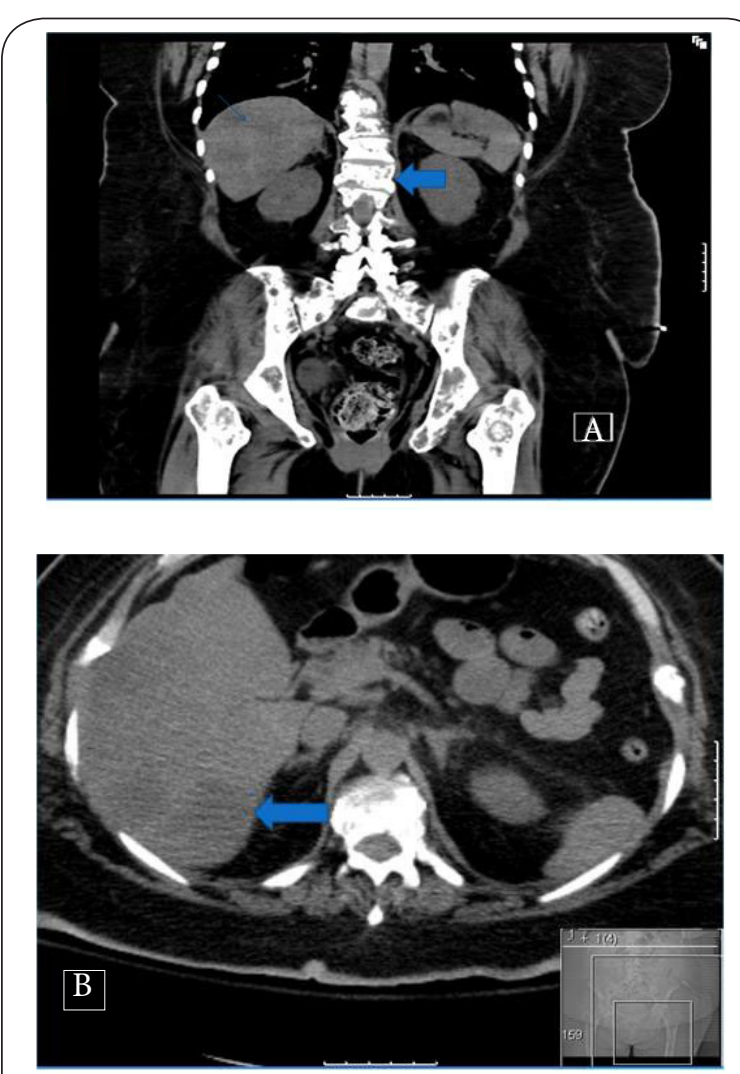

Figure 1. (A) Lytic lesions in lumbar spines see arrow. (B) Multiple hypo dense lesions in the liver, consistent with metastasis.

and severe hyperuricemia associated with acute renal failure.

\section{Treatment}

She was started on normal saline intravenously at a rate of $125 \mathrm{ml} /$ hour, which was increased to $200 \mathrm{cc} /$ hour, metronidazole $500 \mathrm{mg}$ po TID for presumable $C$. Dif. infection. Nephrology and oncology services were consulted. The patient received allopurinol $100 \mathrm{mg}$ orally daily for 5 days, and one dose of rasburicase $4 \mathrm{mg}$ IV. The patient gradually felt better. Her symptoms improved, both diarrhea and vomiting resolved, and her kidney function improved markedly, creatinine dropped to 9.9 (from 13), BUN became 154, and uric acid dropped to 13.1 (from 27.9). We stopped the metronidazole when the results of stool C. Dif. toxins were negative. We discussed with the patient and family the advanced stage of the disease, and the need for compliance with cancer care and follow up. The patient declined chemotherapy. She was discharged to have a return follow up as an outpatient, and was given a prescription of allopurinol $100 \mathrm{mg}$ daily.

\section{Discussion}

Tumor lysis syndrome (TLS) is a medical emergency that occurs in patients with certain cancers and is caused by the rapid and massive breakdown of tumor cells, either spontaneously, or after the initiation of cytoreductive therapy. The rapid release of intracellular contents causes hyperuricemia, hyperkalemia, hyperphosphatemia, and secondary hypocalcemia. These metabolic abnormalities can lead to significant morbidity, putting patients at risk of severe clinical consequences that include acute kidney injury (AKI), cardiac arrhythmias, pulmonary edema, fluid overload, seizures, and even death [1].

\section{Pathophysiology}

Although it can occur spontaneously, TLS is associated most commonly with rapid cell breakdown after initiation of chemotherapy and the consequent massive release of intracellular contents that overwhelms the body's homeostatic and excretory mechanisms. Potassium is stored primarily in the intracellular compartment, and its rapid and excessive release into the extracellular compartment can lead to hyperkalemia, cardiac arrhythmia, and possibly sudden death. Similarly, hyperphosphatemia results from massive release of intracellularly stored phosphate. It can lead to secondary hypocalcaemia and deposition of calcium phosphate crystals in the kidney [1].

Tumors most frequently associated with TLS are clinically aggressive non-Hodgkin lymphomas (NHLs) and acute lymphoblastic leukemia (ALL), particularly Burkitt lymphoma/leukemia [2,3,4-8]. Other hematologic malignancies that are commonly associated with TLS include anaplastic large cell lymphoma, T-cell or B-cell precursor ALL, acute myeloid leukemia (AML), chronic lymphocytic leukemia (CLL), and plasma cell disorders, including multiple myeloma and isolated plasmacytomas $[2,9,10-14]$. TLS has been rarely described after treatment of some nonhematologic solid tumors $[15,16]$. These include breast cancer [17-19], small cell carcinoma (mostly involving the lung) [15,17], neuroblastoma [17], germ cell tumors $[15,20]$, medulloblastoma [15], sarcoma [15,21], ovarian cancer [22,23], squamous cell carcinoma of the vulva [24], metastatic colorectal cancer [25], urothelial cancer [26], gastrointestinal stromal tumors [27], melanoma [15], and hepatocellular carcinoma [15].

\section{Spontaneous TLS}

Spontaneous acute renal failure associated with marked hyperuricemia prior to the initiation of therapy has been described in lymphomas and acute leukemias $[4,10,28,29]$. It usually occurs in patients with bulky, rapidly proliferating, treatment-responsive tumors and has been described in inflammatory breast cancer [30]. The actual incidence of this syndrome is difficult to ascertain. Interestingly, spontaneous TLS is associated with hyperuricemia but frequently without hyperphosphatemia [10]. It has been postulated that rapidly growing neoplasms with high cell turnover rates produce high serum uric acid levels through rapid nucleoprotein turnover but that the 
tumor is able to reutilize released phosphorus for resynthesis of new tumor cells. In contrast, TLS after chemotherapy is due to cell destruction in the absence of reuptake of phosphorus, and thus, hyperphosphatemia [10].

\section{Clinical manifestations of TLS}

The symptoms associated with TLS largely reflect the associated metabolic abnormalities (hyperkalemia, hyperphosphatemia, and hypocalcaemia). These symptoms may include nausea, vomiting, diarrhea, anorexia, lethargy, edema, fluid overload, hematuria, congestive heart failure, cardiac dysrhythmias, seizures, muscle cramps, tetany, syncope, and possibly sudden death [30]. Although these symptoms are multifactorial and may occur before the start of chemotherapy, they are observed more commonly within 12 to 72 hours after the initiation of cytoreductive therapy. Complications resulting from TLS can compromise the efficacy or further administration of chemotherapy [31]. Urinalysis classically shows many uric acid crystals or amorphous urates in acidic urine, but is occasionally relatively normal due to lack of output from the obstructed nephrons.

\section{Management of TLS}

Tumor lysis syndrome management requires the initiation of preventive measures in high-risk patients prior to cancer treatment, as well as the prompt initiation of supportive care for patients who develop acute tumor lysis syndrome during treatment. Patients with evidence of pretreatment acute tumor lysis syndrome should be started immediately on appropriate therapy, with cancer treatment withheld if possible until all parameters are corrected. It would be important to identify high-risk patients before treatment by assessing the extent of tumor burden, histopathologic findings, and renal function. Cancer patients who present with, or develop TLS during therapy should receive intensive nursing care with continuous cardiac monitoring and measurement of electrolytes, creatinine, and uric acid every four to six hours [32]. Effective management involves the combination of treating specific electrolyte abnormalities, and/or acute renal failure, and the use of allopurinol, a xanthine oxidase inhibitor administered to reduce the conversion of nucleic acid byproducts to uric acid in order to prevent urate nephropathy and subsequent oliguric renal failure [33]. It is usually given orally at $600 \mathrm{mg}$ daily for prophylaxis and 600-900 mg daily (up to a maximum of $500 \mathrm{mg} / \mathrm{m}^{2}$ daily) for treatment of tumor lysis syndrome. Patients unable to take oral medications can be given intravenous allopurinol.

On October 16, 2009, the Food and Drug Administration (FDA) granted approval for the use of rasburicase (recombinant urate oxidase) for the initial management of plasma uric acid levels in adult patients with leukemia lymphoma, and solid tumor malignancies who are receiving anticancer therapy expected to result in tumor lysis and subsequent elevation of plasma uric acid. Urate oxidase is an enzyme involved in purine metabolism and is responsible for the oxidation of uric acid to allantoin, which is shown to be 5-10 times more soluble in urine than uric acid [34]. This enzyme is found in most mammals, but not in humans, because of a nonsense mutation in the coding region of the gene during hominoid evolution [35]. Rasburicase is isolated as a cDNA clone from A. flavus and is expressed in Aspergillus flavus the yeast strain Saccharomyces cerevisiae [36]. Rasburicase can be used in cases of TLS when uric acid levels cannot be lowered sufficiently by standard approaches [37]. The advantages of rasburicase are its rapid onset of action and its unique mechanism of action. It lowers preexisting uric acid and prevents the further accumulation of uric acid, therefore, minimizing the risk of tumor lysis syndrome. On the other hand, allopurinol does not have a lowering effect on preexisting uric acid and has a delayed onset of action [37]. Rasburicase is administered at a dose of 0.15 or $0.2 \mathrm{mg} / \mathrm{kg}$ per dose for 5 days. Dosing beyond 5 days or administration of more than 1 course is not recommended by the manufacturer [37]. It is contraindicated in glucose-6-phosphate dehydrogenase (G-6-PD) deficiency and pregnancy [37].

In addition to the above measures, attempting to wash out the obstructing uric acid crystals with a loop diuretic and intravenous fluids is frequently needed, and the appropriate use of renal replacement therapy may become necessary [38]. Indications for hemodialysis include: severe oliguria or anuria, persistent hyperkalemia, hyperphosphatemiainduced symptomatic hypocalcaemia [38].

\section{Conclusion}

Tumor lysis syndrome (TLS) is an oncologic emergency that is caused by massive tumor cell lysis. It can occur after initiation of cytotoxic therapy, but also can occur spontaneously. TLS should be included in the differential diagnosis of patients with breast cancer who present with acute kidney injury even if they did not receive chemotherapy.

\section{Competing interests}

The authors declare that they have no competing interests.

Publication history

Received: 29-Jan-2013 Accepted: 01-Feb-2013

Published: 09-Feb-2013

\section{References}

1. Abu-Alfa A K and Younes A: Tumor lysis syndrome and acute kidney injury: evaluation, prevention, and management. Am J Kidney Dis 2010, 55:S1-13; quiz S14-9. | Article | PubMed

2. Hande $K R$ and Garrow G C: Acute tumor lysis syndrome in patients with high-grade non-Hodgkin's lymphoma. Am J Med 1993, 94:133-9. | Article | PubMed

3. Wossmann W, Schrappe M, Meyer U, Zimmermann M and Reiter A: Incidence of tumor lysis syndrome in children with 
advanced stage Burkitt's lymphoma/leukemia before and after introduction of prophylactic use of urate oxidase. Ann Hematol 2003, 82:160-5. | Article | PubMed

4. Tsokos G C, Balow J E, Spiegel R J and Magrath I T: Renal and metabolic complications of undifferentiated and lymphoblastic lymphomas. Medicine (Baltimore) 1981, 60:218-29. | Article | PubMed

5. Yang $\mathrm{H}$, Rosove $\mathrm{M} \mathrm{H}$ and Figlin $\mathrm{R}$ A: Tumor lysis syndrome occurring after the administration of rituximab in lymphoproliferative disorders: high-grade non-Hodgkin's lymphoma and chronic lymphocytic leukemia. Am J Hematol 1999, 62:247-50. | Article | PubMed

6. Jensen M, Winkler U, Manzke O, DiehI V and Engert A: Rapid tumor lysis in a patient with B-cell chronic lymphocytic leukemia and lymphocytosis treated with an anti-CD20 monoclonal antibody (IDEC-C2B8, rituximab). Ann Hematol 1998, 77:89-91. | Article | PubMed

7. Jabr F I: Acute tumor lysis syndrome induced by rituximab in diffuse large B-cell lymphoma. Int J Hematol 2005, 82:312-4. | Article | PubMed

8. Linck D, Basara N, Tran V, Vucinic V, Hermann S, Hoelzer $D$ and Fauser A A: Peracute onset of severe tumor lysis syndrome immediately after $4 \mathrm{~Gy}$ fractionated TBI as part of reduced intensity preparative regimen in a patient with T-ALL with high tumor burden. Bone Marrow Transplant 2003, 31:935-7. | Article | PubMed

9. Montesinos P, Lorenzo I, Martin G, Sanz J, Perez-Sirvent M L, Martinez D, Orti G, Algarra L, Martinez J, Moscardo F, de la Rubia J, Jarque I, Sanz G and Sanz M A: Tumor lysis syndrome in patients with acute myeloid leukemia: identification of risk factors and development of a predictive model. Haematologica 2008, 93:67-74. | Article | PubMed

10. Kjellstrand C M, Cambell D C, 2nd, von Hartitzsch B and Buselmeier T J: Hyperuricemic acute renal failure. Arch Intern Med 1974, 133:349-59. I Article I PubMed

11. Hussain K, Mazza J J and Clouse L H: Tumor lysis syndrome (TLS) following fludarabine therapy for chronic lymphocytic leukemia (CLL): case report and review of the literature. Am $J$ Hematol 2003, 72:212-5. | Article | PubMed

12. Fassas A B, Desikan K R, Siegel D, Golper T A, Munshi N C, Barlogie $B$ and Tricot $G$ : Tumour lysis syndrome complicating high-dose treatment in patients with multiple myeloma. $\mathrm{Br}$ J Haematol 1999, 105:938-41. | Article | PubMed

13. Berenson J R, Yang H H, Vescio R A, Nassir Y, Mapes R, Lee $S$ $P$, Wilson J, Yellin O, Morrison B, Hilger J and Swift R: Safety and efficacy of bortezomib and melphalan combination in patients with relapsed or refractory multiple myeloma: updated results of a phase 1/2 study after longer follow-up. Ann Hematol 2008, 87:623-31. I Article I PubMed

14. Sezer O, Vesole D H, Singhal S, Richardson P, Stadtmauer E, Jakob C, Boral A L, Esseltine D L and Mehta J: Bortezomibinduced tumor lysis syndrome in multiple myeloma. Clin Lymphoma Myeloma 2006, 7:233-5. | Article | PubMed

15. Baeksgaard L and Sorensen J B: Acute tumor lysis syndrome in solid tumors--a case report and review of the literature. Cancer Chemother Pharmacol 2003, 51:187-92. | Article | PubMed

16. Gemici C: Tumour lysis syndrome in solid tumours. Clin Oncol (R Coll Radiol) 2006, 18:773-80. | Article | PubMed

17. Kalemkerian G P, Darwish B and Varterasian M L: Tumor lysis syndrome in small cell carcinoma and other solid tumors. Am J Med 1997, 103:363-7. | Article | PubMed
18. Drakos P, Bar-Ziv J and Catane R: Tumor lysis syndrome in nonhematologic malignancies. Report of a case and review of the literature. Am J Clin Oncol 1994, 17:502-5. | Article | PubMed

19. Rostom A Y, El-Hussainy G, Kandil A and Allam A: Tumor lysis syndrome following hemi-body irradiation for metastatic breast cancer. Ann Oncol 2000, 11:1349-51. | Article | PubMed

20. Pentheroudakis G, O'Neill V J, Vasey P and Kaye S B: Spontaneous acute tumour lysis syndrome in patients with metastatic germ cell tumours. Report of two cases. Support Care Cancer 2001, 9:554-7. | Article | PubMed

21. Gold J E, Malamud S C, LaRosa F and Osband M E: Adoptive chemoimmunotherapy using ex vivo activated memory T-cells and cyclophosphamide: tumor lysis syndrome of a metastatic soft tissue sarcoma. Am J Hematol 1993, 44:42-7. | Article | PubMed

22. Bilgrami S F and Fallon B G: Tumor lysis syndrome after combination chemotherapy for ovarian cancer. Med Pediatr Oncol 1993, 21:521-4. | Article | PubMed

23. Chan J K, Lin S S, McMeekin D S and Berman M L: Patients with malignancy requiring urgent therapy: CASE 3. Tumor lysis syndrome associated with chemotherapy in ovarian cancer. J Clin Oncol 2005, 23:6794-5. | Article | PubMed

24. Shamseddine A I, Khalil A M and Wehbeh M H: Acute tumor lysis syndrome with squamous cell carcinoma of the vulva. Gynecol Oncol 1993, 51:258-60. | Article | PubMed

25. Oztop I, Demirkan B, Yaren A, Tarhan O, Sengul B, Ulukus C, Akin D, Sen M, Yilmaz U and Alakavuklar M: Rapid tumor lysis syndrome in a patient with metastatic colon cancer as a complication of treatment with 5-fluorouracil/leucoverin and irinotecan. Tumori 2004, 90:514-6. | PDF I PubMed

26. Lin C J, Lim K H, Cheng Y C, Chen H H and Wu C J: Tumor lysis syndrome after treatment with gemcitabine for metastatic transitional cell carcinoma. Med Oncol 2007, 24:455-7. | Article I PubMed

27. Pinder E M, Atwal G S, Ayantunde A A, Khan S, Sokal M, McCulloch T and Parsons S L: Tumour Lysis Syndrome Occurring in a Patient with Metastatic Gastrointestinal Stromal Tumour Treated with Glivec (Imatinib Mesylate, Gleevec, STI571). Sarcoma 2007, 2007:82012. | Article | PubMed Abstract | PubMed Fulltext

28. Jasek A M and Day H J: Acute spontaneous tumor lysis syndrome. Am J Hematol 1994, 47:129-31. | Article | PubMed

29. Hsu H H and Huang C C: Acute spontaneous tumor lysis in anaplastic large T-cell lymphoma presenting with hyperuricemic acute renal failure. Int J Hematol 2004, 79:4851. | Article | PubMed

30. Sklarin N T and Markham M: Spontaneous recurrent tumor lysis syndrome in breast cancer. Am J Clin Oncol 1995, 18:713. | Article | PubMed

31. Coiffier B, Altman A, Pui C H, Younes A and Cairo M S: Guidelines for the management of pediatric and adult tumor lysis syndrome: an evidence-based review. J Clin Oncol 2008, 26:2767-78. | Article | PubMed

32. Howard S C, Jones D P and Pui C H: The tumor lysis syndrome. N Engl J Med 2011, 364:1844-54. | Article | PubMed Abstract | PubMed Fulltext

33. Mahmoud H H, Leverger G, Patte C, Harvey E and Lascombes $\mathrm{F}$ : Advances in the management of malignancy-associated 
hyperuricaemia. Br J Cancer 1998, 77 Suppl 4:18-20. | Article | PubMed Abstract | PubMed Fulltext

34. Brogard J M, Coumaros D, Franckhauser J, Stahl A and Stahl J: Enzymatic uricolysis: a study of the effect of a fungal urateoxydase. Rev Eur Etud Clin Biol 1972, 17:890-5. | Article | PubMed

35. Yeldandi A V, Yeldandi V, Kumar S, Murthy C V, Wang X D, Alvares K, Rao M S and Reddy J K: Molecular evolution of the urate oxidase-encoding gene in hominoid primates: nonsense mutations. Gene 1991, 109:281-4. | Article | PubMed

36. Leplatois P, Le Douarin B and Loison G: High-level production of a peroxisomal enzyme: Aspergillus flavus uricase accumulates intracellularly and is active in Saccharomyces cerevisiae. Gene 1992, 122:139-45. | Article | PubMed

37. Yim B T, Sims-McCallum R P and Chong P H: Rasburicase for the treatment and prevention of hyperuricemia. Ann Pharmacother 2003, 37:1047-54. | Article | PubMed

38. Coiffier B, Altman A, Pui C H, Younes A and Cairo M S: Guidelines for the management of pediatric and adult tumor lysis syndrome: an evidence-based review. J Clin Oncol 2008, 26:2767-78. | Article | PubMed

\section{Citation:}

Elfar A, Teleb M, Rodriguez C and Nahleh Z: Spontaneous tumor lysis syndrome in breast cancer: a case report and discussion. journal of Cancer Therapeutics and Research 2013, 2:9. http://dx.doi.org/10.7243/2049-7962-2-9 